\title{
Dun-52.5. 33
}

\section{DISCLAIMER}

This report was prepared as an account of work sponsored by an agency of the United States Government. Neither the United States Government nor any agency thereof, nor any of their employees, makes any warranty, express or implied, or assumes any legal liability or responsibility for the accuracy, completeness, or usefulness of any information, apparatus, product, or process disclosed, or represents that its use would not infringe privately owned rights. Reference herein to any specific commercial product, process, or service by trade name, trademark, manufacturer, or otherwise does not necessarily constitute or imply its endorsement, recommendation, or favoring by the United States Government or any agency thereof. The views and opinions of authors expressed herein do not necessarily state or reflect those of the United States Government or any agency thereof.

\section{Cover Sheet for a Hanford Historical Document Released for Public Availability}

Released 1994

Prepared for the U.S. Department of Energy under Contract DE-AC06-76RLO 1830

Pacific Northwest Laboratory Operated for the U.S. Department of Energy by Battelle Memorial Institute 


\section{DISCLAIMER}

Portions of this document may be illegible in electronic image products. Images are produced from the best available original document. 


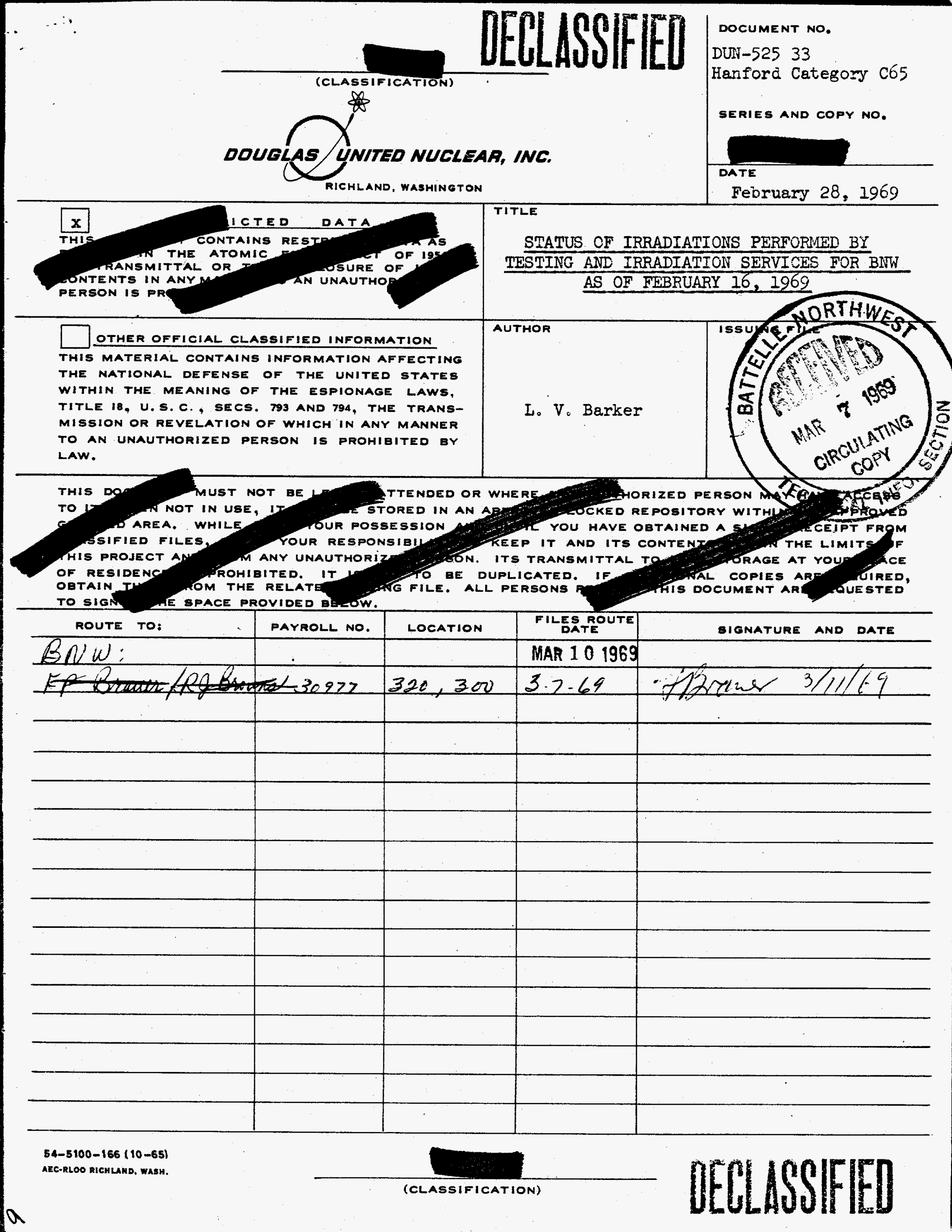




\section{DECLLSSSFFED}

DUN-525 33

Hanford Category C-65

This document contains restricted detes defined in the Atomic Enemes

Its transmitto conten to an unauthorized This document consists of prohibited.

DISTRIBUTION

1. IV Barker

2. DUN File

3. DUN Record

Battelle-Northwest

4. FP Brauer/RJ Brouns

5. TT Claudson

6. WJ Gray

7. RD Leggett

STATUS OF IRRADIATIONS PERFORMED BY TESTING AND IRRADIATION SERVICES FOR BNW AS OF FEBRUARY I6, 1969

L. V. Barker

February 28, 1969

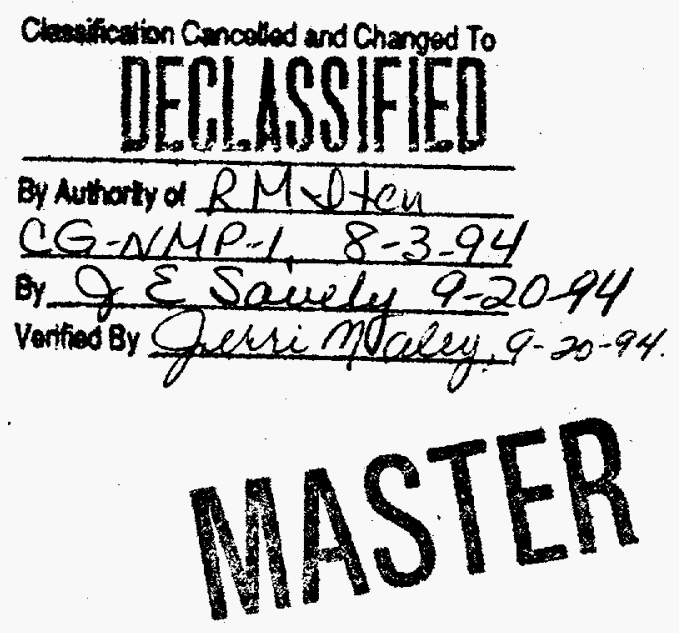


This report itemizes the Irradiations performed by Testing and Irradiation Services for Battelle-Northwest. It lists the material being irradiated, awaiting disposition and material shipped during the report period. An explanation of the various columns in the report follows:

\section{Identification}

a. TISR No. - This is the official number assigned by Testing and Irradiation Services to an experiment for purposes of identification.

b. Request Number - Customer experiment identification number, if any.

c. Material - The principle target material, if known, abbreviated or its chemical symbol in capital letters.

d. Plece Number - Individual sample or assembly identification.

Operating Time (Days) - The number of full-power operating days the sample has been exposed.

CMK Absorbed - The total cmk absorbed by the sample. Does not include the facility.

Charge Date - Date and time sample charged into reactor or date and time reactor reached one-half equilibrium level if sample was charged during a reactor outage.

Location

a. Facility - Test facility or process tube and reactor containing the experiment.

b. Test Hole Position - Sample position in-reactor relative to a process tube.

c. Position Factor - Location of sample in relation to front-to-rear cosine flux distribution. (This factor only applies to front-to-rear irradiations.)

NOTE: The actual shape may deviate from a cosine curve due to local perturbations. The actual shape will be provided upon request.

Exposure to Date - NVT - The total thermel neutron exposure per square centimeter received to date. This number is calculated from facility unperturbed flux and reactor operating time.

Discharge, Date and Time - Date and time samples were removed from the active portion of the reactor or date and time reactor was down to one-half equilibrium level if sample was discharged during a reactor outege.

Shipping Date - Date samples were shipped to the customer or otherwise disposed of.

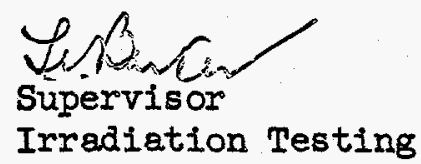

IV Barker/k 


$\left[\begin{array}{ll}n \\ : 11 \\ :\end{array}\right.$

NV N

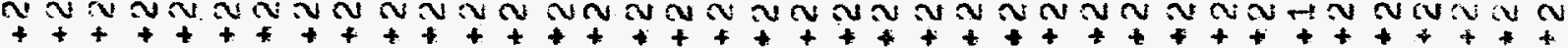

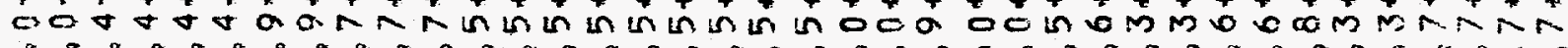

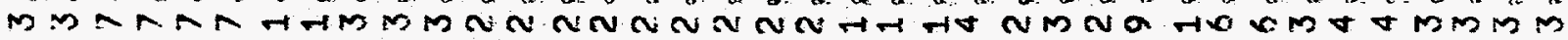

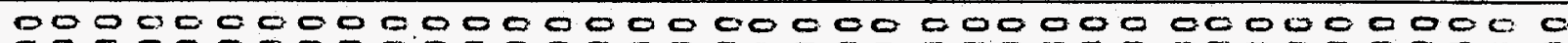
CEE

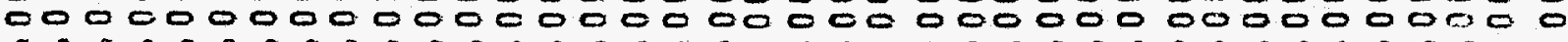

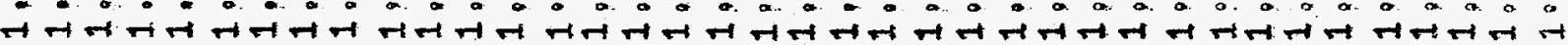
का แ

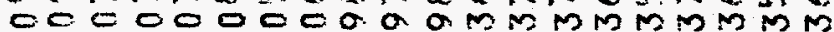

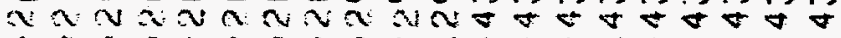

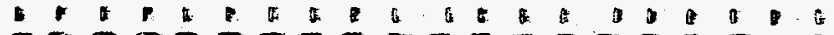

$\operatorname{lin} \mathrm{m} N \mathrm{O}$ $M R M N$ H $N \propto N \Omega$ N $x<x: x$

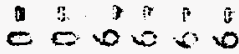

4

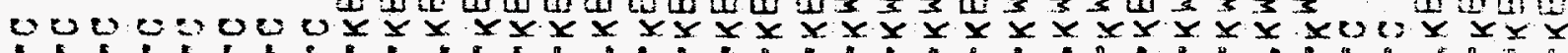

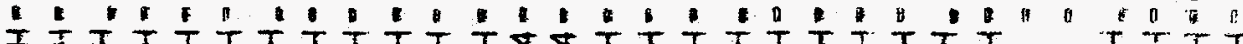

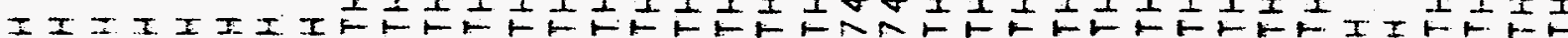
FF-1FE

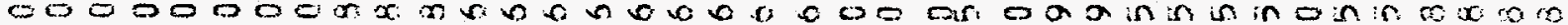

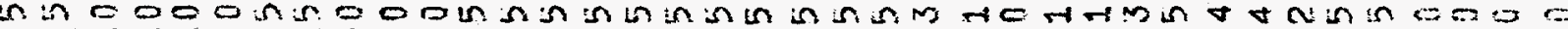

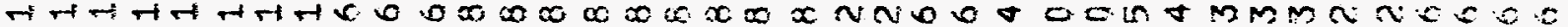
NNHHA HNO

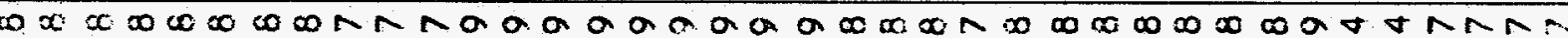

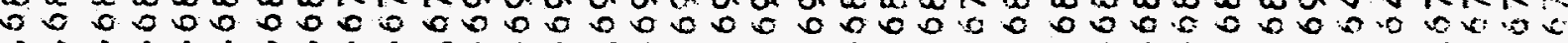

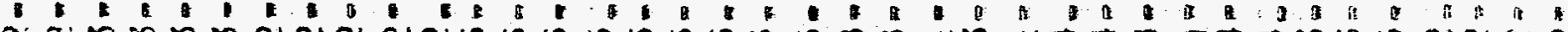
N

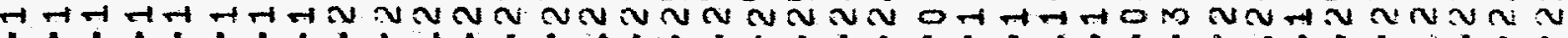
N

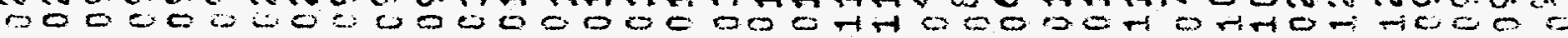

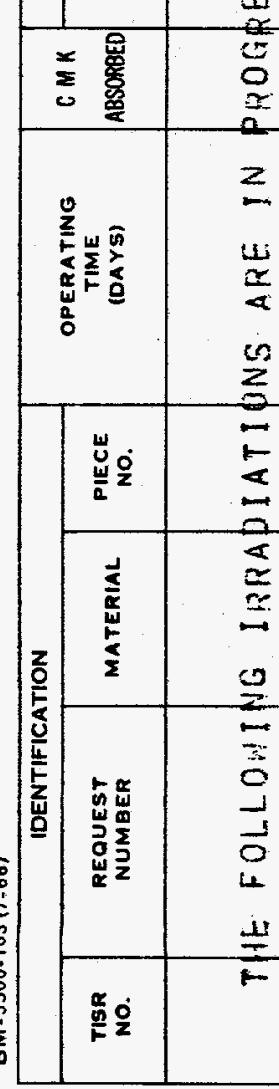

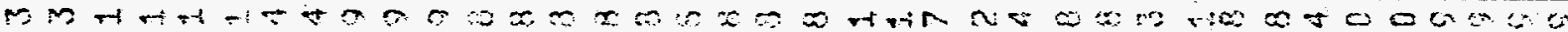

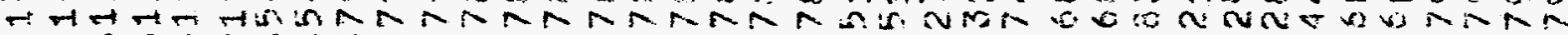
c

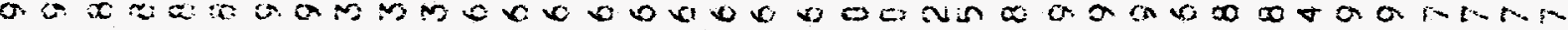

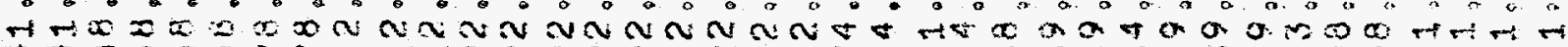

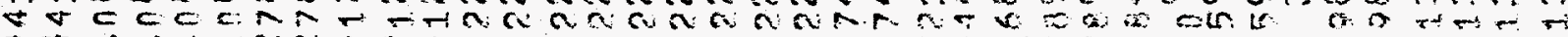
a r.t:

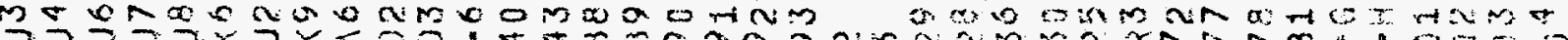

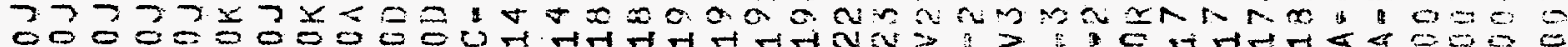

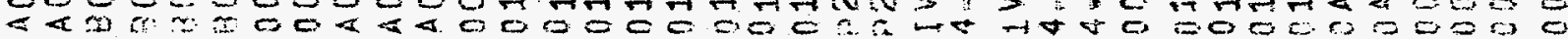

IIIIIIIIIIIIIIIIIIIIT

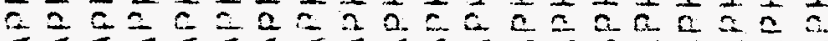

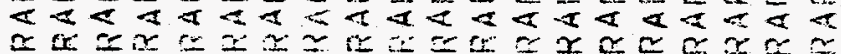

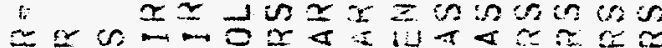

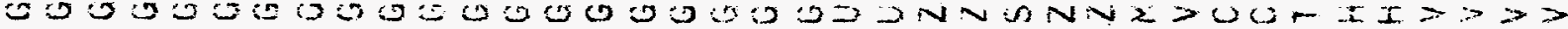

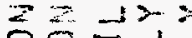

$u=>00 \Leftrightarrow \vec{E}$

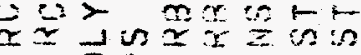

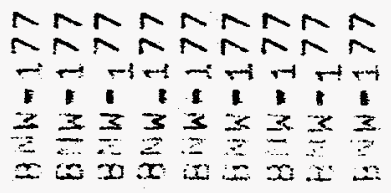

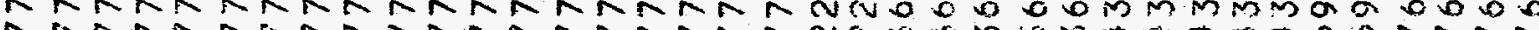

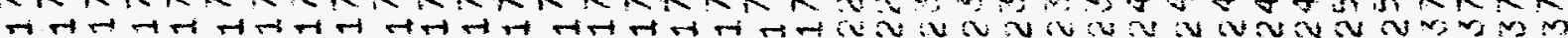




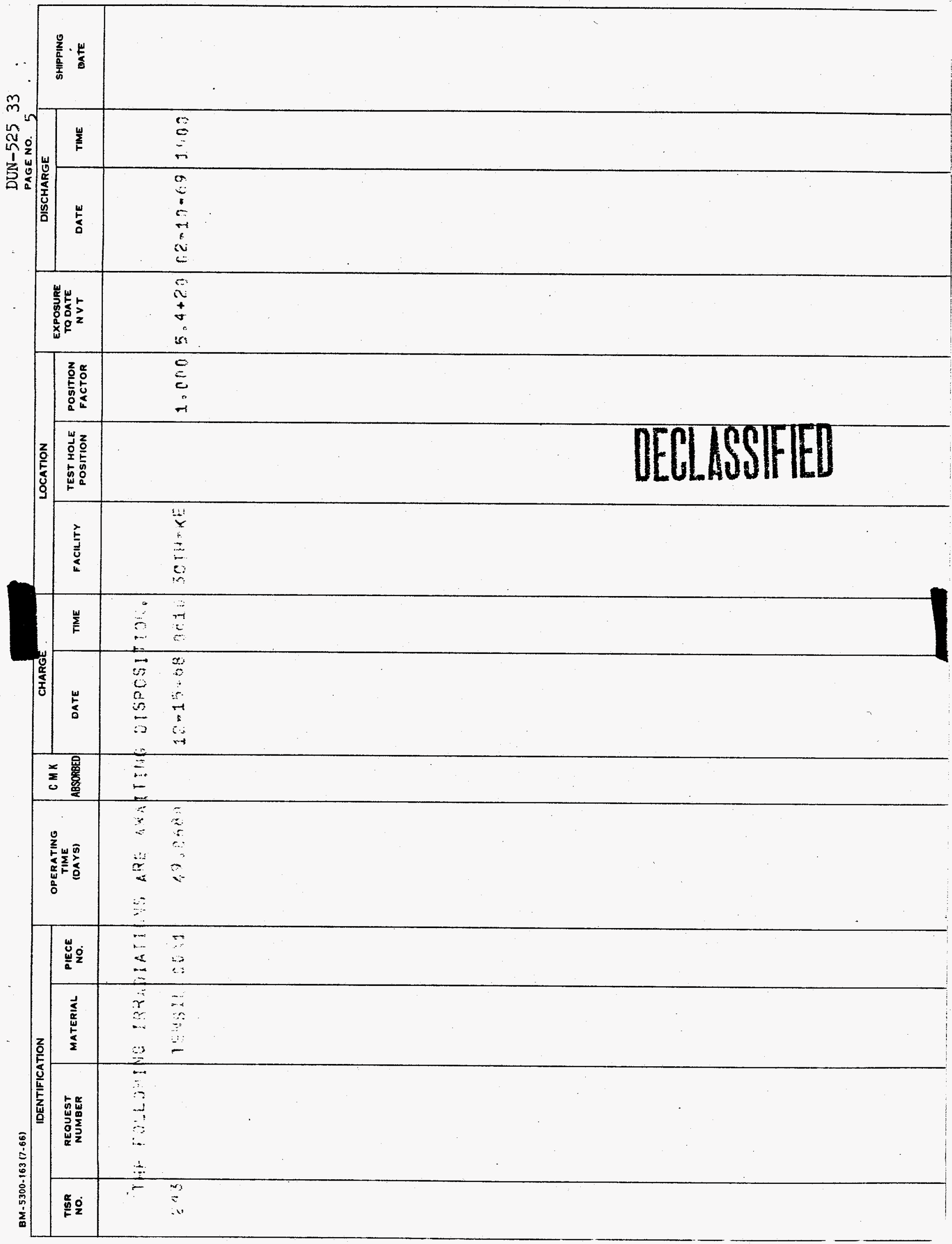




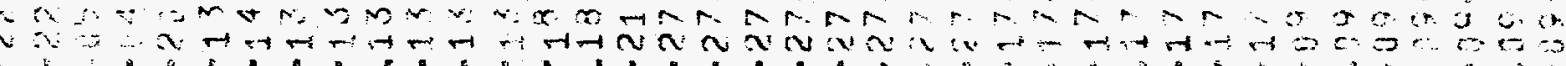

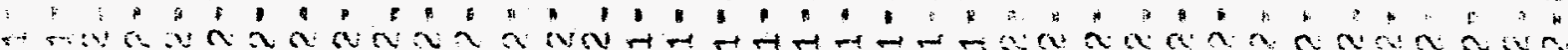

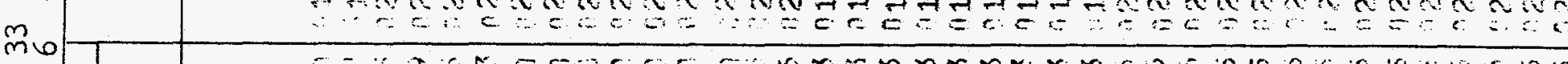

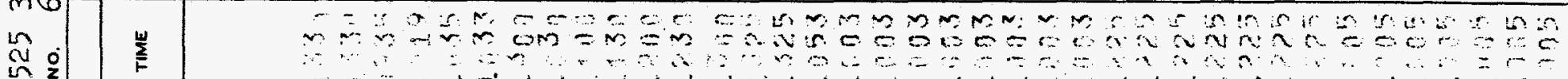

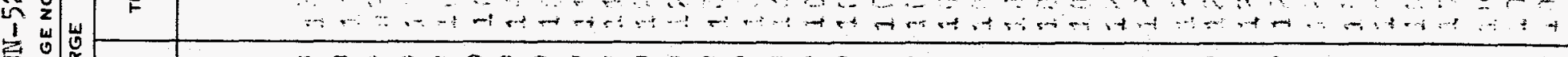

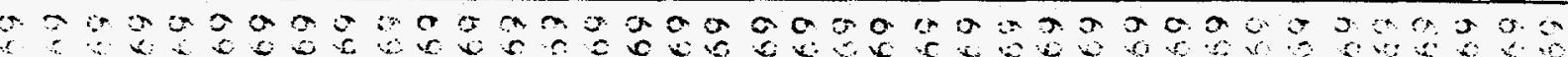

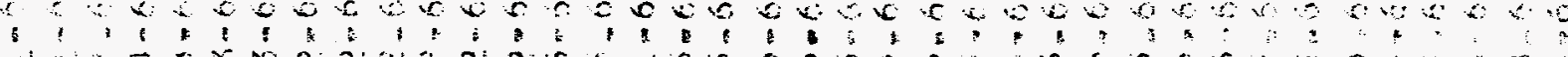

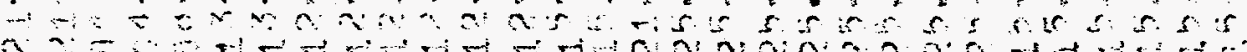

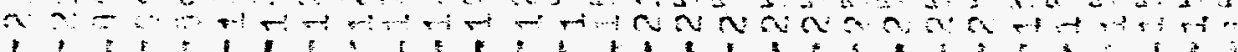
$\because \cdots$ 7. 造款

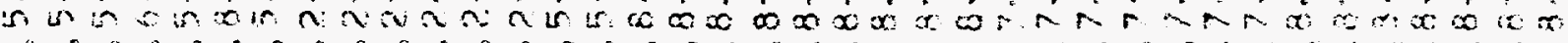

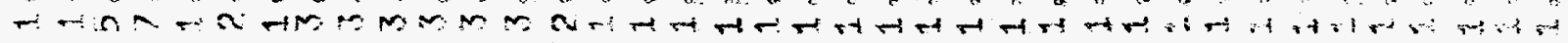

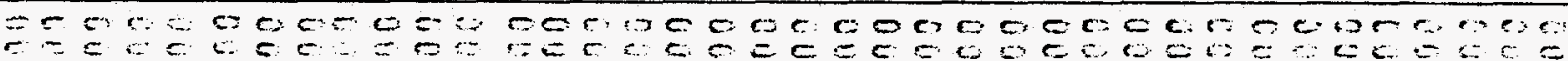

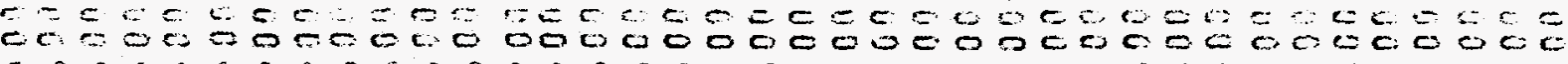

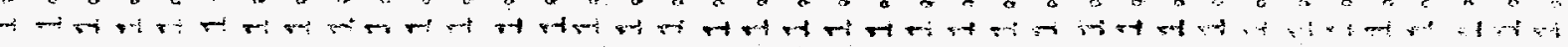

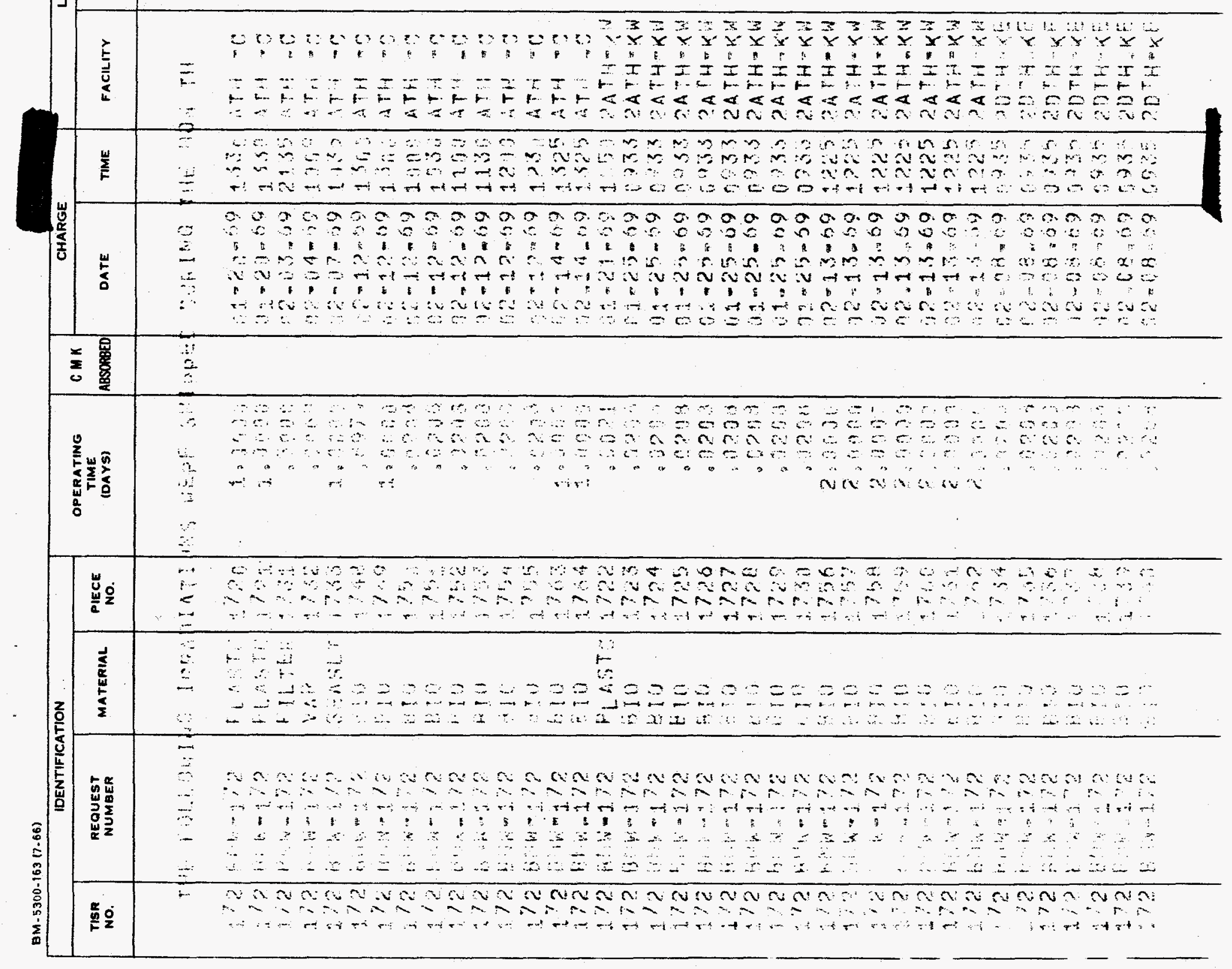




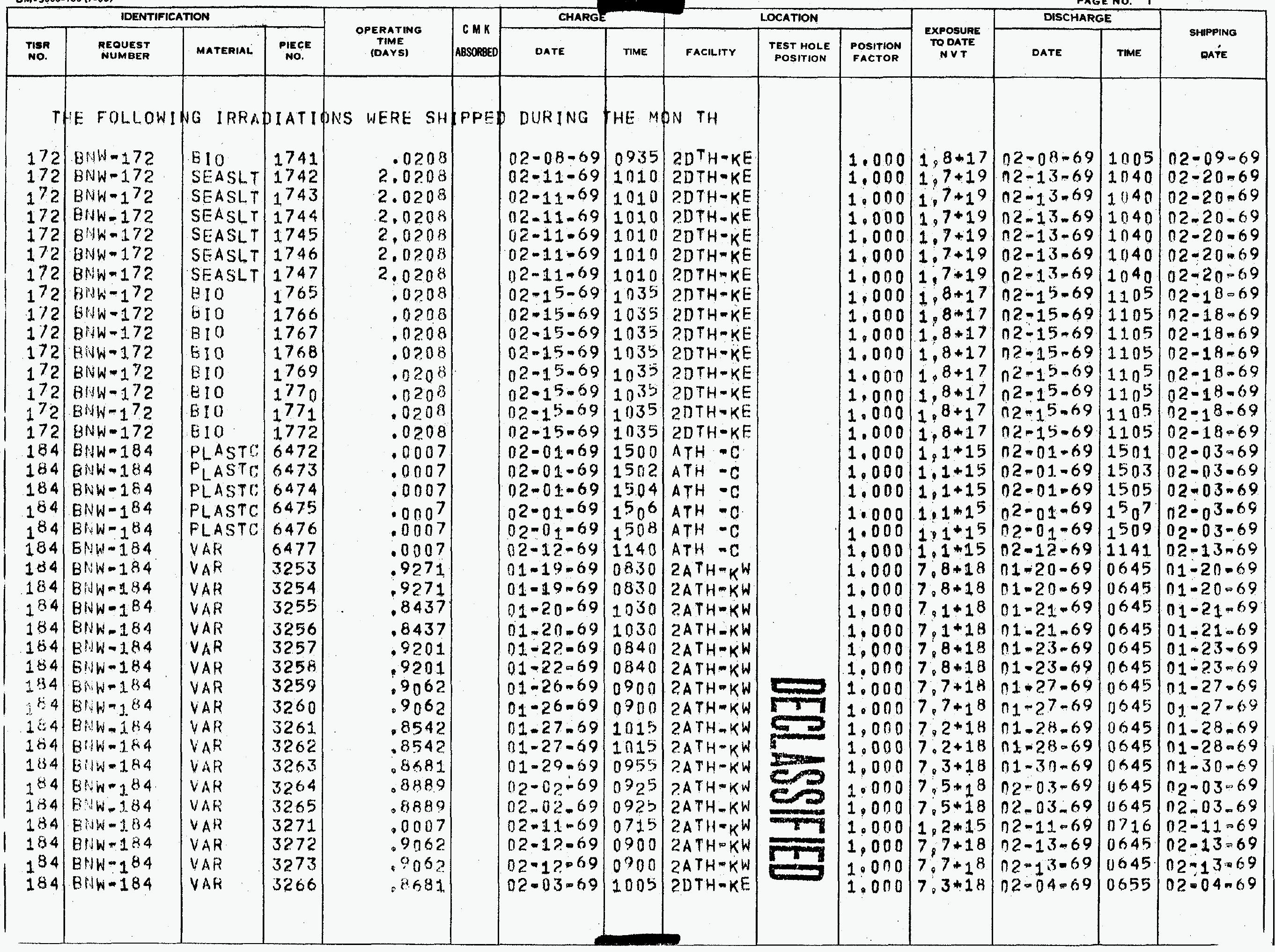


